\title{
Cluster-Former: Clustering-based Sparse Transformer for Question Answering
}

\author{
Shuohang Wang Luowei Zhou Zhe Gan Yen-Chun Chen \\ Yuwei Fang Siqi Sun Yu Cheng Jingjing Liu \\ Microsoft Corporation \\ \{shuowa, luowei.zhou, zhe.gan, yen-chun.chen\}@microsoft.com \\ \{yuwfan, siqi.sun, yu.cheng, jingjl\}@microsoft.com
}

\begin{abstract}
Transformer has become ubiquitous in the deep learning field. One of the key ingredients that destined its success is the self-attention mechanism, which allows fully-connected contextual encoding over input tokens. However, despite its effectiveness in modeling short sequences, self-attention suffers when handling inputs with extreme long-range dependencies, as its complexity grows quadratically w.r.t. the sequence length. Therefore, long sequences are often encoded by Transformer in chunks using a sliding window. In this paper, we propose Cluster-Former, a novel clusteringbased sparse Transformer to perform attention across chunked sequences. The proposed framework is pivoted on two unique types of Transformer layer: Sliding-Window Layer and Cluster-Former Layer, which encode local sequence information and global context jointly and iteratively. This new design allows information integration beyond local windows, which is especially beneficial for question answering (QA) tasks that rely on long-range dependencies. Experiments show that ClusterFormer achieves state-of-the-art performance on several major QA benchmarks.
\end{abstract}

\section{Introduction}

Long-range contextual understanding has proven critical in many natural language processing (NLP) tasks. For example, the relevant context for correctly answering an open-domain question can arch over thousands of words (Chen et al., 2017). Encoding long sequences via deep neural networks, however, has remained an expensive and challenging task due to high demand on training time and GPU memory. Traditional sequence modeling methods (Hochreiter and Schmidhuber, 1997) encode long sequences in a chronological order, which suffers high latency. In the place of sequential encoding, recent models such as Trans- former (Vaswani et al., 2017) use simultaneous selfattention over the entire input instead, which has been successfully adopted in many NLP tasks such as textual entailment (Devlin et al., 2019), dependency parsing (Zhou and Zhao, 2019), and summarization (Lewis et al., 2019). A caveat with Transformer though is that building full connections over long sequences translates to quadratic growth on memory demand and computational complexity w.r.t. sequence length.

One way to efficiently encode long sequences is to first chunk a sequence into much shorter ones with a sliding window, then build connections between the shorter sequences (Figure 1(a)). For example, Child et al. (2019), Beltagy et al. (2020) and Zaheer et al. (2020) apply sparse attention to chunked sequences in hand-designed patterns in order to gather information from the chunks (Figure 1(b)). Choi et al. (2017) and Wang et al. (2019) first use a simpler model to filter chunked sequences, then process selected sequences with fully-connected self-attention. Rae et al. (2019) makes use of the shared memory of chunked sequences to build connections between them. However, these methods cannot encode long-range dependencies with as much flexibility or accuracy as fully-connected self-attention, due to their dependency on handdesigned patterns.

Recently, several studies (Kitaev et al., 2020; Tay et al., 2020a) propose to further improve the sparse attention mechanism by hashing or sorting the hidden states into different buckets (Figure 1(c)). These works mainly explore tasks with relatively short sequences, such as sentence-level machine translation, where the number of hashing vectors is relatively small (less than 16 in $\mathrm{Ki}$ taev et al. (2020)), allowing randomly initialized hashing vectors to hash hidden states into correct buckets. However, how to use hashing-based attention in the context of long sequences (e.g.,, up to 


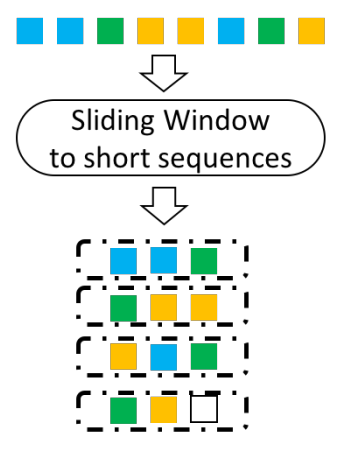

(a) Sliding window

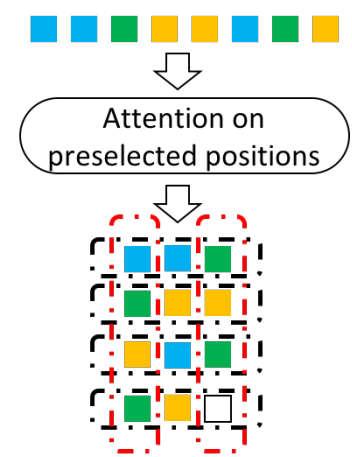

(b) Sparse Attention

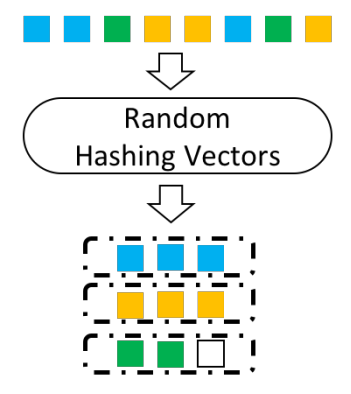

(c) Locality-Sensitive Hashing

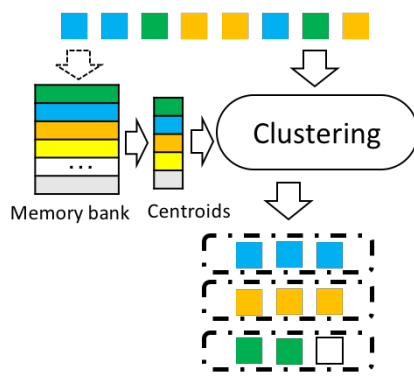

(d) K-Means Clustering

Figure 1: Illustration of different methods for processing long sequences. Each square represents a hidden state. The black-dotted boxes are Transformer layers. (a) is the sliding-window-based method to chunk a long sequence into short ones with window size 3 and stride 2. (b) builds cross-sequence attention based on sliding window over pre-selected positions (red-dotted boxes). (c) hashes the hidden states into different buckets by randomlyinitialized vectors. (d) is our proposed approach to cluster the hidden states. Our final model is a combination of (a) and (d) that processes both local and global context.

thousands of words) is still an unexplored territory.

Our proposed framework for efficient long sequence encoding, Cluster-Former, marries both sliding-window and hashing-based methods to achieve effective local and long-range dependency encoding. Cluster-Former consists of two types of encoding layer. The first one (noted as SlidingWindow Layer) focuses on extracting local information within a sliding window. It applies Transformer to the hidden states of each chunked sequence independently, as shown in Figure 1(a). The other one (noted as Cluster-Former Layer) learns to encode global information beyond the initial chunked sequences. Specifically, we first apply clustering to the input hidden states so that similar hidden states are assigned to the same cluster, as shown in Figure 1(d). The clustered and sorted input is then divided uniformly into chunks, each encoded by a Transformer layer. Note that to make model training more efficient, the cluster centroids are not computed online but updated periodically (every epoch or a few epochs). We accumulate the hidden states from the layer prior to the Cluster-Former layer in a memory bank, and apply the K-Means algorithm to form cluster centroids during each update cycle. Compared to previously discussed sparse attention based on pre-selected positions (Figure 1(b)) or randomly-initialized hashing vectors (Figure 1(c)), experimental results show that our method can encode dependency across chunked sequences more effectively.

Our contributions can be summarized as follows. (i) We propose Cluster-Former, a novel approach to capturing long-range dependencies more effectively than locality-sensitive hashing method. (ii) We propose a new Transformer-based framework to process long sequences by combining SlidingWindow and Cluster-Former layers to extract both local and global contextual information. (iii) Our model achieves the best performance on question answering datasets of Natural Questions (long answer), SearchQA, and Quasar-T.

\section{Related Work}

Efficient Transformers With Transformer models growing larger and larger, how to handle longer sequences arises as a critical challenge. Many works have been proposed to improve the computational and memory efficiency of Transformers, including Sparse Transformer (Child et al., 2019), Set Transformer (Lee et al., 2019), Routing Transformer (Roy et al., 2020), Fast Transformer (Vyas et al., 2020), Reformer (Kitaev et al., 2020), Sinkhorn Transformer (Tay et al., 2020a), Longformer (Beltagy et al., 2020), ETC (Ainslie et al., 2020), Synthesizer (Tay et al., 2021), Performer (Choromanski et al., 2020), Linformer (Wang et al., 2020), Linear Transformer (Katharopoulos et al., 2020), and BigBird (Zaheer et al., 2020). Tay et al. (2020b) provided an excellent literature survey on this emerging topic. Our method falls into the setting of learnable sparse-attention patterns.

Among all these works, our method is closer to Set Transformer (Lee et al., 2019), Routing Transformer (Roy et al., 2020), and Fast Trans- 


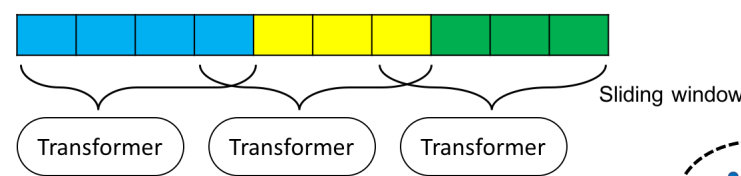

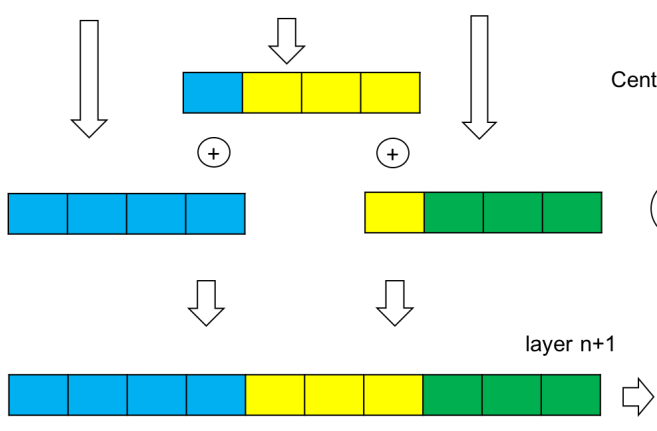

(a) Transformer in sliding window

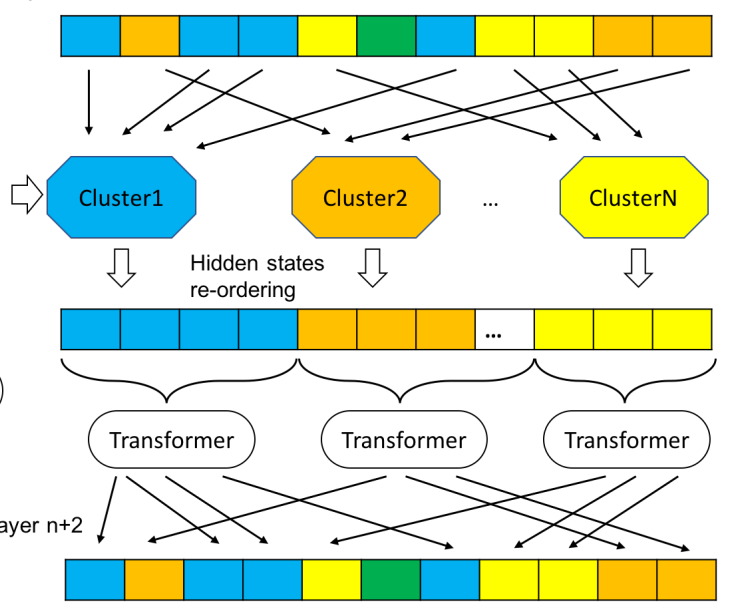

(b) Cluster-Former

Figure 2: An overview of the proposed Transformer layer. (a) Sliding-Window layer over a sequence. (b) ClusterFormer layer over clustered hidden states from the output of (a). Cluster centroids are periodically updated based on the memory bank of the hidden states in the corresponding layer.

former (Vyas et al., 2020), which all use cluster centroids to learn patterns. However, we target at solving a different task, question answering. And it also leads to a significant different framework to encode a short question with a long context, other than a single long sequence, such as language modeling task. Moreover, our cluster centroids are updated in a very different way by periodical centroids update with K-Means on memory bank, other than memory-based centroids (Lee et al., 2019), exponentially moving centroids (Roy et al., 2020), or online clustering (Vyas et al., 2020).

Long Sequence in Question Answering For tasks such as open-domain question answering (Chen et al., 2017), a large volume of documents or paragraphs is usually retrieved to infer the answer, yielding extremely long context content. Despite the fact that state-of-the-art NLP models are capable of extracting answers amid complex context, they still struggle with extremely long input sequences. Recent advances that advocate the use of large-scale pre-trained models (Lewis et al., 2019; Liu et al., 2019; Lan et al., 2020) for question answering make this problem more prominent, due to tremendous memory consumption. To process long sequences, the most widely-used method is to first use a lightweight model to filter out redundant text, then use sliding-window-based approaches to encode the remaining sequences with a more sophisticated model. Chen et al. (2017) integrated bi-gram features into Information Retrieval (IR) methods to retrieve related documents more accurately. Wang et al. (2018) trained a paragraph selector using as the reward whether the entire system can obtain the correct answer or not. Asai et al. (2020) trained a recurrent retriever to select paragraphs for multi-hop question answering. Izacard and Grave (2021) proposed to fuse local encoded information into a decoder for answer generation. Besides the above methods, directly applying Efficient Transformers to process long sequences in question answering is another option. In this paper, we focus on this direction by directly training our Cluster-Former on the long context without using lightweight model for context filtering.

\section{Proposed Approach}

The proposed framework to handle long sequences is pivoted on two types of Transformer layer: (i) Sliding-Window Layer; and (ii) Cluster-Former Layer. The former focuses on encoding local sequence information, while the latter is on encoding global context and always built on top of the former layer. An overview of the two layers is illustrated in Figure 2.

\subsection{Sliding-Window Layer}

Despite that our focus is on capturing long-range dependencies for global context, local information also plays a critical role for knowledge propagation. Therefore, in the lower section of our network, we adopt the traditional sliding-window encoding mechanism. A sliding window segments 
a long sequence $X$ into short, overlapping ones with window size $l$ and stride $m$, as illustrated in Figure 2(a). Note that in this paper, we focus on question answering tasks, for which we concatenate the question $Q$ with each sequence chunked from the document:

$$
\mathbf{H}_{k}^{0}=[\mathbf{Q} ; \mathbf{X}[m \times k:(m \times k+l)]],
$$

where $\mathbf{Q} \in \mathbb{R}^{q \times d}$ denotes question embeddings given a QA task, $q$ is the number of tokens in the question, and $\mathbf{X} \in \mathbb{R}^{x \times d}$ is the embeddings for all context, $x$ is the number of tokens in context. $k$ is the ID of the chunked sequence, $l$ is the window size, and $m$ is the stride of the sliding window. $\left[i d x_{1}: i d x_{2}\right]$ indicates selecting rows between the index of $i d x_{1}$ and $i d x_{2}$ of the matrix. $[\cdot ; \cdot]$ means concatenating the matrices along the row. We first use Transformer to encode each sequence in sliding window as follows:

$$
\mathbf{H}_{k}^{n+1}=\text { Transformer }\left(\mathbf{H}_{k}^{n}\right),
$$

where $\mathbf{H}_{k}^{n+1} \in \mathbb{R}^{(q+l) \times d}$ is the output of Transformer on the $k$-th sequence in the $n$-th layer, while it is not the final output of the $n$-th layer. As we expect the neighbouring sequences to share useful information in hidden states as well, we always set $m<l$ to allow overlapping between sequences. We use the mean values of the Transformer hidden states at the overlapped tokens between windows as final outputs. To merge the representations from the $(k-1)$-th sequence:

$$
\begin{aligned}
& \mathbf{H}_{k}^{n+1}[q: q+l-m] \quad+=\mathbf{H}_{k-1}^{n+1}[q+m: e n d] \\
& \mathbf{H}_{k}^{n+1}[q: q+l-m] \quad /=2,
\end{aligned}
$$

and merge representations from $(k+1)$-th sequence:

$$
\begin{aligned}
& \mathbf{H}_{k}^{n+1}[q+m: e n d] \quad+=\mathbf{H}_{k+1}^{n+1}[q: q+l-m], \\
& \mathbf{H}_{k}^{n+1}[q+m: e n d] \quad /=2,
\end{aligned}
$$

where $+=$ is to add matrices in-place and / $=$ is to divide a matrix by a scalar value in-place. The merged hidden states $\mathbf{H}_{k}^{n+1} \in \mathbb{R}^{(q+l) \times d}$ are the final outputs of the $n$-th layer. If the next layer is Cluster-Former, the output hidden states in this layer $\mathbf{H}_{k}^{n+1}$ will be saved into memory bank for computing the cluster centroids.

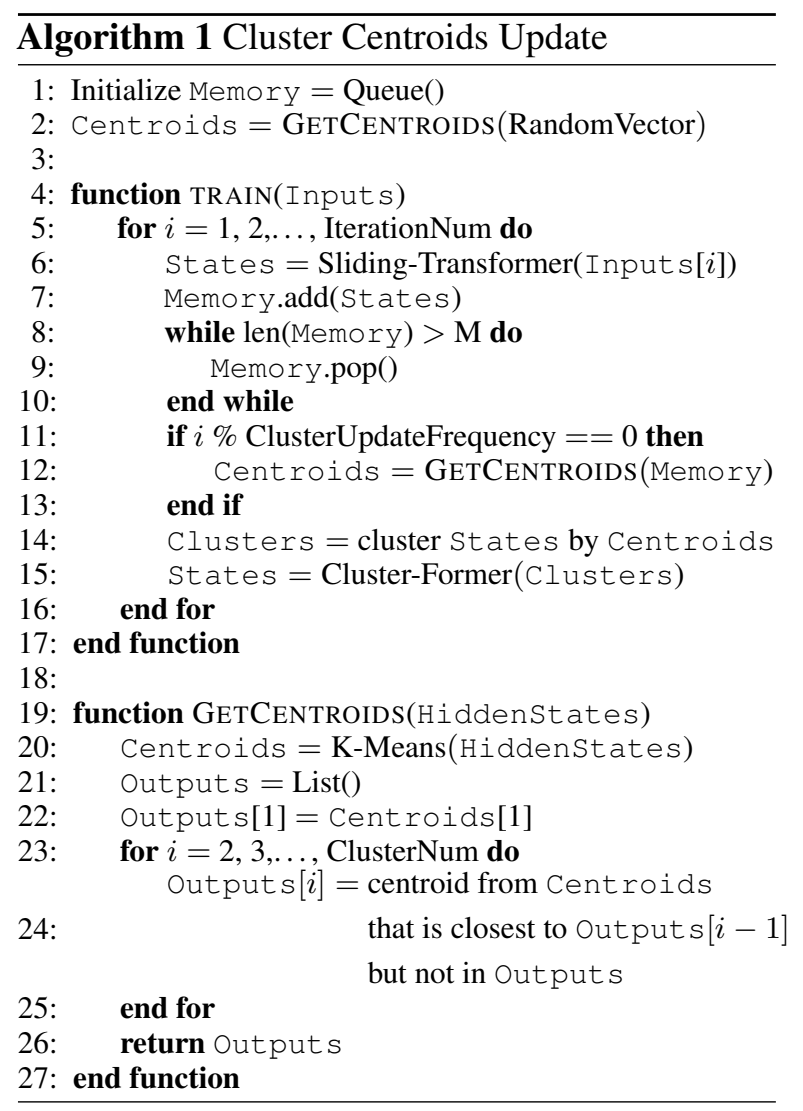

\subsection{Cluster-Former Layer}

We introduce a Cluster-Former layer to add global representational power to Transformer beyond sliding windows. An in-depth visualization of the layer is illustrated in Figure 2(b).

The input of the Cluster-Former layer comes from the hidden states of the prior layer (in our case a Sliding-Window layer). After merging the overlaps between sequence chunks, the input of this layer is defined as:

$$
\overline{\mathbf{H}}^{n}=\left[\mathbf{H}_{0}^{n}[0: q+m] ; \ldots ; \mathbf{H}_{k}^{n}[0: q+m]\right],
$$

where $\overline{\mathbf{H}}^{n} \in \mathbb{R}^{(q\lceil x / m\rceil+x) \times d}$ is the hidden states to cluster, $x$ is the number of tokens in the context.

As the hidden states with larger cosine similarity are more likely to have higher attention weights, we build sparse self-attention only on the hidden states in the same cluster. In this work, we use K-Means as the chosen clustering method for simplicity. More advanced clustering algorithms have the potential of yielding better performance. Since running K-Means on the fly in each training iteration is computationally expensive, we decide to recompute the cluster centroids with low frequency (every epoch or a few epochs).

In addition, to avoid dramatic changes in the cluster centroids due to limited hidden state inputs, 
we maintain a memory bank for the most recent hidden states. The entire procedure is depicted in Algorithm 1. Once we compute the cluster centroids, we can directly use them for hidden state clustering as follows:

$$
\mathbf{v}^{n}=\operatorname{argmax}\left(\frac{\mathbf{H}^{n}\left(\mathbf{C}^{n}\right)^{T}}{\left\|\mathbf{H}^{n}\right\|_{2}\left\|\mathbf{C}^{n}\right\|_{2}}\right),
$$

where $\mathbf{C}^{n} \in \mathbb{R}^{p \times d}$ are the cluster centroids for layer $n$, and $p$ is the pre-defined number of clusters. The function $\operatorname{argmax}(\cdot)$ performs on the last dimension and assigns all the input hidden states into different clusters based on the max value of cosine similarity between the hidden states and cluster centroids. $\mathbf{v}^{n} \in \mathbb{R}^{(q\lceil x / m\rceil+x)}$ is the assigned cluster IDs of all the input hidden states.

Since the number of hidden states in different clusters can vary substantially, padding them to the maximum length for Transformer training will significantly increase the computational time. To make the extraction of global context more efficient, we greedily pick the cluster centroids based on the nearest neighbour (measured by cosine similarity) as shown in the function GETCENTROIDS in Algorithm 1. Thus, the hidden states with similar cluster IDs are also close to each other. Then, we can directly sort the cluster IDs of hidden states and uniformly chunk the hidden states (same window size and stride $m$ ):

$$
\begin{aligned}
\mathbf{u}^{n} & =\operatorname{argsort}\left(\mathbf{v}^{n}\right), \\
\mathbf{a}_{k}^{n} & =\mathbf{u}^{n}[m k: m(k+1)], \\
\mathbf{E}_{k}^{n} & =\mathbf{H}^{n}\left[\mathbf{a}_{k}^{n}\right],
\end{aligned}
$$

where the function $\operatorname{argsort}(\cdot)$ is to obtain the indexes of input values sorted in order (same values sorted by the corresponding position of hidden states). $\mathbf{a}_{k}^{n} \in \mathbb{R}^{m}$ is the chunked indexes of the hidden states. $\mathbf{E}_{k}^{n} \in \mathbb{R}^{m \times d}$ is the $k$-th clustered hidden states, and we will run Transformer on top of it to build the connection beyond the words in the initial sliding window as follows:

$$
\mathbf{E}_{k}^{n+1}=\text { Transformer }\left(\mathbf{E}_{k}^{n}\right) .
$$

After updating the hidden states, we map them back to the order before clustering:

$$
\begin{aligned}
\overline{\mathbf{H}}^{n+1} & =\left[\mathbf{E}_{0}^{n+1} ; \mathbf{E}_{1}^{n+1} ; \ldots ; \mathbf{E}_{K}^{n+1}\right], \\
\overline{\mathbf{a}}^{n} & =\left[\mathbf{a}_{0}^{n} ; \mathbf{a}_{1}^{n} ; \ldots ; \mathbf{a}_{K}^{n}\right], \\
\overline{\mathbf{H}}^{n+1}\left[\overline{\mathbf{a}}^{n}\right] & =\operatorname{clone}\left(\overline{\mathbf{H}}^{n+1}\right),
\end{aligned}
$$

\begin{tabular}{lcccc}
\hline & \#train & \#test & med & max \\
\hline Quasar-T & $29 \mathrm{k}$ & $3 \mathrm{k}$ & $2.8 \mathrm{k}$ & $8.2 \mathrm{k}$ \\
SearchQA & $100 \mathrm{k}$ & $27 \mathrm{k}$ & $2.5 \mathrm{k}$ & $4.9 \mathrm{k}$ \\
NQ & $292 \mathrm{k}$ & $8 \mathrm{k}$ & $6.3 \mathrm{k}$ & $128 \mathrm{k}$ \\
\hline
\end{tabular}

Table 1: Statistics of Question Answering datasets. \#train: number of questions in the training set. \#test: number of questions in the test set. med: median length of the context. max: max length of the context.

where $\overline{\mathbf{H}}^{n+1}$ is the final output hidden state of this layer and has the same word order as the input $\overline{\mathbf{H}}^{n}$. In experiments, we stack these two types of layer interchangeably to capture both global and local context efficiently.

\section{Experiments}

\subsection{Datasets}

We evaluate our proposed approach on multiple question answering benchmarks. The statistics of all the datasets are summarized in Table 1 .

- Quasar-T ${ }^{1}$ (Dhingra et al., 2017): The goal of this task is to answer open-domain questions from Trivia Challenge. All the passages harvested through information retrieval can be used to answer questions. The task requires the model to generate answers in phrases. The evaluation metric on this dataset is based on Exact Match and F1 score of the bag-of-words matching. Our evaluation tool ${ }^{2}$ comes from the SQuAD dataset.

- SearchQA ${ }^{3}$ (Dunn et al., 2017): The setting of this dataset is the same as Quasar-T, except that the questions are sourced from Jeopardy! instead.

- Natural Questions ${ }^{4}$ (Kwiatkowski et al., 2019): This task aims to answer questions based on a given Wikipedia document, and has two settings. (i) Long answer: select a paragraph that can answer the question based on the Wikipedia document if any. (ii) Short answer: extract an answer phrase from the document if the document contains the answer. As the given document may not contain answer, we can either predict an answer or predict no answer. The evaluation metric on this dataset is the F1 score, where true positives are exactly correct answers, false positives are

\footnotetext{
${ }^{1}$ https://github.com/bdhingra/quasar

${ }^{2}$ https://rajpurkar.github.io/SQuAD-explorer/

${ }^{3}$ https://github.com/nyu-dl/dl4ir-searchQA

${ }^{4}$ https://ai.google.com/research/NaturalQuestions
} 


\begin{tabular}{lcccc}
\hline & Quasar-T & SearchQA & NQ(long) & NQ(short) \\
& EM/F1 & EM/F1 & F1 & F1 \\
\hline R3 (Wang et al., 2018) & $35.3 / 41.7$ & $49.0 / 55.3$ & - & - \\
DECAPROP (Tay et al., 2018) & $38.6 / 46.9$ & $62.2 / 70.8$ & - & - \\
DS-QA (Lin et al., 2018) & $42.2 / 49.3$ & $58.8 / 64.5$ & - & - \\
Multi-passage BERT (Wang et al., 2019) & $51.1 / 59.1$ & $65.1 / 70.7$ & - & - \\
DrQA (Chen et al., 2017) & $37.7 / 44.5$ & $41.9 / 48.7$ & 46.1 & 35.7 \\
DecAtt + DocReader (Kwiatkowski et al., 2019) & - & - & 54.8 & 31.4 \\
BERT $_{\text {joint }}$ (Alberti et al., 2019) & - & - & 64.7 & 52.7 \\
BERT $_{\text {wwm + SQuAD2 (Pan et al., 2019) }}$ & - & - & 68.2 & 57.2 \\
RikiNet-RoBERTa (Liu et al., 2020) & - & - & 75.3 & $\mathbf{5 9 . 3}$ \\
\hline Sliding Window & $52.9 / 62.8$ & $65.8 / 73.2$ & 75.3 & 56.4 \\
Sparse Attention (Child et al., 2019) & $52.1 / 62.0$ & $64.7 / 71.7$ & 74.5 & 56.1 \\
Locality-Sensitive Hashing (Kitaev et al., 2020) & $53.2 / 62.9$ & $66.0 / 73.5$ & 75.5 & 56.4 \\
\hline Cluster-Former (\#C=64) & $53.3 / 63.3$ & $67.0 / 74.2$ & 76.3 & 56.7 \\
Cluster-Former (\#C=256) & $53.6 / 63.5$ & $67.5 / 74.5$ & 76.3 & 56.7 \\
Cluster-Former (\#C=512) & $\mathbf{5 4 . 0 / 6 3 . 9}$ & $\mathbf{6 8 . 0 / 7 5 . 1}$ & $\mathbf{7 6 . 5}$ & 57.1 \\
\hline
\end{tabular}

Table 2: Results on Quasar-T, SearchQA test sets and NQ dev set. \#C: number of clusters.

\begin{tabular}{lcccccc}
\hline & \multicolumn{3}{c}{ Long Answer } & \multicolumn{3}{c}{ Short Answer } \\
& F1 & Precision & Recall & F1 & Precision & Recall \\
\hline BigBird-ETC-large (Zaheer et al., 2020) & 77.8 & 77.5 & $\mathbf{7 8 . 1}$ & 57.9 & 63.7 & 53.0 \\
RikiNet (Liu et al., 2020) & 76.1 & 78.1 & 74.2 & $\mathbf{6 1 . 3}$ & $\mathbf{6 7 . 6}$ & 56.1 \\
\hline Cluster-Former (Ours) & $\mathbf{7 8 . 0}$ & $\mathbf{7 8 . 5}$ & 77.5 & 60.9 & 62.1 & $\mathbf{5 9 . 8}$ \\
\hline
\end{tabular}

Table 3: Results on Natural Questions (NQ) leaderboard (test set). We show two published results here from over 40 submissions. Our model achieves No.1 for long answer and No.4 for short answer.

incorrect answer predictions, and false negatives are incorrect "no answer" predictions. As the test set is hidden, we split $5 \%$ of the training set for validation, and use the original validation set for testing. We use the official tool from the dataset to evaluate our models. We also submit our best model to the leaderboard.

\subsection{Implementation Details}

All the models are trained on 8 Nvidia V100 GPUs. For clustering, we adopt "Yinyang kmeans" (Ding et al., 2015) ${ }^{5}$ which takes less than 5 seconds for clustering in all our experiment settings. We set the memory size for clustering $M=100,000$ in Algorithm 1. Based on our experiments, it makes little difference for memory banks with $50 \mathrm{k}$ and $100 \mathrm{k}$, update cycles with 1 iteration or half iteration. We use cluster centroids that perform the best on the validation set for test set experiments. As

\footnotetext{
${ }^{5}$ https://github.com/src-d/kmcuda
}

the cluster-centroid is offline computed, the inference time is the same as the sliding-window-based method. We initialize our models with RoBERTalarge (Liu et al., 2019). As the number of position embeddings of RoBERTa is limited to 512, we cannot assign different position embeddings to all tokens. Instead, we assign the same position embeddings to each chunked sequence.

The majority of our model is made up of SlidingWindow Layers, as the local information is essential for QA tasks. We adopt the proposed ClusterFormer Layer in layers 15 and 20 to further capture long-range information. We set the sliding window size $l$ to 256 , stride $m$ to 224 , and change the number of clusters in $\{64,256,512\}$ to analyze its impact on the final performance. We prepend a special token to the beginning of all the given/retrieved paragraphs and directly concatenate all the paragraphs as the final context sequence. Due to memory constraints, we set the max length to be 5000 


\begin{tabular}{ccccc}
\hline & 3 & 4 & 5 & 6 \\
\hline 8 & $55.7 / 65.0$ & $55.6 / 64.4$ & $54.7 / 64.3$ & $55.4 / 64.6$ \\
12 & $55.1 / 64.9$ & $55.8 / 65.0$ & $\mathbf{5 6 . 1 / 6 5 . 4}$ & $55.4 / 64.6$ \\
16 & $55.6 / 65.0$ & $55.2 / 64.7$ & $55.1 / 64.6$ & $54.8 / 64.1$ \\
20 & $54.8 / 64.2$ & $55.4 / 64.8$ & $55.1 / 64.6$ & - \\
\hline
\end{tabular}

Table 4: Experiments on Quasar-T dev dataset. $a \in$ $\{3,4,5,6\}$ and $b \in\{8,12,16,20\}$, if the layer number $l \% a==0$ and $l>=b$, we set it as Cluster-Former Layer, otherwise Sliding Window Layer.

during training and 10000 during inference. During dataset finetuning, we use Adam (Kingma and $\mathrm{Ba}, 2015)$ to optimize the model. We set warm-up updates to 2,220, maximal updates to 22,200, learning rate to $5 \times 10^{-5}$, and batch size to 160 . We tune the dropout rate from $\{0.1,0.15,0.2\}$ for all the methods including baselines and report the best results. The model converges in one day for all the QA datasets.

For Quasar-T and SearchQA, we predict the start and end positions of the answer. For Natural Question, we first identify whether the question has short/long answers or not based on the mean values of the first hidden state of all the chunked sequences, $\frac{1}{K} \sum_{k=1}^{K} \mathbf{H}_{k}^{N}[0]$, where $K$ is the number of chunks and $N$ is the number of layers. If answerable, we rank all the candidates for long answer selection, and predict the start and end positions of short answers. Our model submitted to Natural Question Leaderboard ensembled 3 models with 512 clusters, and only these models are firstly trained on SQuAD2.0 and then finetuned on Natural Question dataset.

\subsection{Baselines}

We compare our models with several strong baselines, including:

$\mathbf{R 3}$ (Wang et al., 2018) proposes to use reinforcement learning to jointly train passage ranker and reader. DS-QA (Lin et al., 2018) proposes to first use paragraph selection to filter the noisy data and then trained model on denoised data. Multipassage BERT (Wang et al., 2019) proposes to filter the passages and then merge multiple useful passages into one sequence, which can be encoded by BERT. DrQA (Chen et al., 2017) makes use of attention mechanism across the question and the document for answer phrase extraction. DecAtt and DocReader (Kwiatkowski et al., 2019) is based on a pipeline approach that first uses a simpler model

\begin{tabular}{lcc}
\hline & $\begin{array}{c}\text { Wikitext } \\
\text { ppl }\end{array}$ & $\begin{array}{c}\text { Enwik8 } \\
\text { bpc }\end{array}$ \\
\hline Sliding window & 20.8 & 1.34 \\
Sparse Attention & 20.5 & 1.29 \\
Locality-Sensitive Hashing & 20.8 & 1.33 \\
\hline Cluster-Former (\#C=64) & 20.5 & 1.28 \\
Cluster-Former (\#C=256) & 20.3 & 1.24 \\
Cluster-Former $(\# \mathrm{C}=512)$ & $\mathbf{2 0 . 2}$ & $\mathbf{1 . 2 2}$ \\
\hline
\end{tabular}

Table 5: Results on Language Modeling. \#C: number of clusters; Wikitext: Wikitext-103.

to select long answers and then a reading comprehension model to extract short answers from the long answers. BERT joint $_{\text {(Alberti et al., 2019) }}$ jointly trains short and long answer extraction in a single model rather than using a pipeline approach. BERT $_{\text {wwm }}+$ SQuAD2 (Pan et al., 2019) makes use of multi-task learning to further boost performance. RikiNet-RoBERTa (Liu et al., 2020) proposes a dynamic paragraph dual-attention reader and a multi-level cascaded answer predictor. BigBirdETC (Zaheer et al., 2020) makes use of a sparse attention mechanism to encode long sequences.

We also re-implement several strong baselines which have not been applied to process long context in question answering tasks:

- Sliding Window: The original method is fully made up of Sliding-Window Layers and can only attend to local information. To make a fair comparison among different methods on long-range information collection, we replace several layers of this sliding window baseline with Sparse Attention, Locality-Sensitive Hashing, and ClusterFormer.

- Sparse Attention (Child et al., 2019): This method replaces several layers in the previous baseline by training a Transformer layer across sequences on pre-selected positions. We run this sparse Transformer on all the hidden states in the same position across sequences, so that the output of sparse Transformer can merge the information from different sequences.

- Locality-Sensitive Hashing (Kitaev et al., 2020): This method hashes hidden states into different buckets determined by randomlyinitialized hashing vectors. A Transformer layer is then applied across buckets to build Sparse 


\begin{tabular}{|c|c|}
\hline $\begin{array}{l}\text { Question } \\
\text { Context }\end{array}$ & $\begin{array}{l}\text { Where did the underground railroad start and finish? } \\
\text { The Underground Railroad by artist Charles T. Webber, } 1893 \text { Date Late 1700s - } 1865 \\
\text { Location Northern United States with routes to Canada , Mexico ... }\end{array}$ \\
\hline $\begin{array}{l}\text { Special token } \\
\text { Time }\end{array}$ & $\begin{array}{l}<\mathrm{s}><\mathrm{s}><\mathrm{s}>\text { Island island in the colonies city }<\mathrm{s}><\mathrm{s}><\mathrm{s}>\text { With in the in } . \\
\text { did start and finish } 1893 \text { Date } 17001865 \text { Location Participants Outcome Deaths } 19 \\
1763\end{array}$ \\
\hline $\begin{array}{l}\text { Stopwords } \\
\text { Entity }\end{array}$ & $\begin{array}{l}\text { the the , the },,, \text {, to },,, \text {, the American runaway slaves of free states the },,, \text { it to , a the } \\
\text { Canada Mexico Canada is applied Florida Spanish Railroad Railroad Railroad }\end{array}$ \\
\hline Positions & $49,50,51,52,53,54,55,115,116,168,273,394, \ldots, 6022,6040,6042,6060,6094$ \\
\hline
\end{tabular}

Table 6: An example from Natural Question dataset. The rows in the middle section show the corresponding words of the clustered hidden states, and the bottom row shows the positions of the clustered hidden states. " $<$ s $>$ " refers to start token of long answer candidate.

Attention across the whole sequence. Note that this method cannot be directly used for question answering without adding Sliding-Window layer, as our QA model is initialized by RoBERTa that only has 512 position embeddings.

\subsection{Experimental Results}

State-of-the-Art Results on QA Table 2 and 3 show that our proposed method outperforms several strong baselines, thanks to its ability to encode both local and global information. Cluster-Former with 512 clusters achieves new state-of-the-art results on Quasar-T, SearchQA and Natural Question (long answer).

Effect of Cluster-Former We also test the ability of Cluster-Former on modeling long-range dependencies. Note that Sparse Attention (Child et al., 2019) and Locality-Sensitive Hashing (Kitaev et al., 2020) have never been tested on question answering tasks with long context. For fair comparison, we set the layers 15 and 20 as either Sparse Attention, Locality-Sensitive Hashing or our ClusterFormer, and the left layers are Sliding Window layers.

As shown, Sparse Attention performs worse than our Cluster-Former. The loss may come from the noise introduced by pre-selected positions, the corresponding words of which may not be related. We set the number of hashing vectors in LocalitySensitive Hashing (LSH) to 64, the same as the number of clusters in Cluster-Former. LSH outperforms the baseline slightly on QA and consistently underperforms our Cluster-Former $(\# \mathrm{C}=64)$. Overall, our Cluster-Former performs the best.

Effect of Number of Cluster Centroids We also test the effect of different numbers of cluster centroids $(C)$ on model performance. We observe that the model with 512 clusters works significantly better than the model with 64 clusters on most of the tasks. However, for Natural Questions Long Answer setting, the improvement is marginal. As we mainly rely on the hidden state of special tokens " $<\mathrm{s}>$ " for long answer selection, and the same tokens can be assigned into same chunk more easily even with a smaller number of clusters.

Selection of Cluster-Former Layers We also have an analysis on which layers are better used for Cluster-Former layer. As shown in Table 4, we conduct a hyper-parameter search. And find that it can get better performance with at least one ClusterFormer layers in the middle layer (8-16). The worst results come from only one Cluster-Former layer in the layer of 22 or 23.

Language Modeling Although we focus on QA tasks, to demonstrate the versatility of ClusterFormer, we conduct additional experiments on language modeling using the Wikitext-103 (Merity et al., 2017) and Enwik8 (Mahoney, 2011) benchmarks. All the models are trained from scratch. We set the number of layers to 16 , with 8 heads per layer. Our Cluster-Former Layer is used in layers 11 and 15 as in QA models. We segment long input into short sequences of 3072 tokens, set sliding window size $l$ to 256 , and stride $m$ to 128 . SGD is used for optimizing the models. We set clip threshold of gradients to 0.1 , warm-up updates to 16,000 , maximal updates to 286,000 , dropout rate to 0.3 , learning rate to 0.1 , and batch size to 16. The model will converge in 3 days for all the LM datasets. As shown in Table 5, Cluster-Former outperforms strong state-of-the-art baselines. 


\subsection{Qualitative Analysis}

We perform qualitative analysis on how the hidden states are clustered, by visualizing the corresponding words and positions of the hidden states in Table 6 . From the first row, we can see that the special tokens " $<\mathrm{s}>$ " tend to belong to the same cluster. Note that " $<\mathrm{s}>$ " is the start token of each long answer candidate, and its hidden state is used for final long answer selection. Therefore, Transformer on this cluster can compare across the candidates to make the final prediction.

We further observe that the same types of token are more likely to appear in the same cluster. For example, words from the second row to the forth row cover the topics of time, stopwords, and organization \& geopolitical entities.

Finally, we randomly sample a cluster and list the positions of clustered hidden states in the last row of the table. We find that states in long distance, such as the 50-th and 6060-th states (over 6000 tokens apart), can be in one cluster, which demonstrates the ability of Cluster-Former in detecting long-range dependencies. Further, we observe that states tend to cluster in phrases. For example, we see consecutive positions such as " 49 , $50,51,52,53,54,55$ ”, which likely results from the sliding-window encoding.

\section{Conclusion}

In this paper, we present Cluster-Former, a new method to encode global information for long sequences. We achieve new state of the art on three question answering datasets: Quasar-T, SearchQA, and Natural Questions. Further, we observe that a larger number of clusters in Cluster-Former can lead to better performance on question answering tasks. Cluster-Former is a generic approach, and we believe that it can benefit other NLP tasks that rely on long-range dependencies as well.

\section{References}

Joshua Ainslie, Santiago Ontanon, Chris Alberti, Philip Pham, Anirudh Ravula, and Sumit Sanghai. 2020. Etc: Encoding long and structured data in transformers. In Empirical Methods in Natural Language Processing (EMNLP).

Chris Alberti, Kenton Lee, and Michael Collins. 2019. A bert baseline for the natural questions. arXiv preprint arXiv:1901.08634.

Akari Asai, Kazuma Hashimoto, Hannaneh Hajishirzi, Richard Socher, and Caiming Xiong. 2020. Learn- ing to retrieve reasoning paths over wikipedia graph for question answering. In International Conference on Learning Representations (ICLR).

Iz Beltagy, Matthew E Peters, and Arman Cohan. 2020. Longformer: The long-document transformer. arXiv preprint arXiv:2004.05150.

Danqi Chen, Adam Fisch, Jason Weston, and Antoine Bordes. 2017. Reading Wikipedia to answer opendomain questions. In Association for Computational Linguistics (ACL).

Rewon Child, Scott Gray, Alec Radford, and Ilya Sutskever. 2019. Generating long sequences with sparse transformers. arXiv preprint arXiv:1904.10509.

Eunsol Choi, Daniel Hewlett, Jakob Uszkoreit, Illia Polosukhin, Alexandre Lacoste, and Jonathan Berant. 2017. Coarse-to-fine question answering for long documents. In Association for Computational Linguistics (ACL).

Krzysztof Choromanski, Valerii Likhosherstov, David Dohan, Xingyou Song, Jared Davis, Tamas Sarlos, David Belanger, Lucy Colwell, and Adrian Weller. 2020. Masked language modeling for proteins via linearly scalable long-context transformers. arXiv preprint arXiv:2006.03555.

Jacob Devlin, Ming-Wei Chang, Kenton Lee, and Kristina Toutanova. 2019. Bert: Pre-training of deep bidirectional transformers for language understanding. In North American Chapter of the Association for Computational Linguistics (NAACL).

Bhuwan Dhingra, Kathryn Mazaitis, and William W Cohen. 2017. Quasar: Datasets for question answering by search and reading. arXiv preprint arXiv:1707.03904.

Yufei Ding, Yue Zhao, Xipeng Shen, Madanlal Musuvathi, and Todd Mytkowicz. 2015. Yinyang kmeans: A drop-in replacement of the classic kmeans with consistent speedup. In International conference on machine learning (ICML).

Matthew Dunn, Levent Sagun, Mike Higgins, V Ugur Guney, Volkan Cirik, and Kyunghyun Cho. 2017. Searchqa: A new q\&a dataset augmented with context from a search engine. arXiv preprint arXiv:1704.05179.

Sepp Hochreiter and Jürgen Schmidhuber. 1997. Long short-term memory. Neural Computation.

Gautier Izacard and Edouard Grave. 2021. Leveraging passage retrieval with generative models for open domain question answering. In European Chapter of the Association for Computational Linguistics (EACL).

Angelos Katharopoulos, Apoorv Vyas, Nikolaos Pappas, and François Fleuret. 2020. Transformers are rnns: Fast autoregressive transformers with linear attention. arXiv preprint arXiv:2006.16236. 
Diederik P Kingma and Jimmy Ba. 2015. Adam: A method for stochastic optimization. In International Conference on Learning Representations (ICLR).

Nikita Kitaev, Łukasz Kaiser, and Anselm Levskaya. 2020. Reformer: The efficient transformer. In International Conference on Learning Representations (ICLR).

Tom Kwiatkowski, Jennimaria Palomaki, Olivia Redfield, Michael Collins, Ankur Parikh, Chris Alberti, Danielle Epstein, Illia Polosukhin, Jacob Devlin, Kenton Lee, et al. 2019. Natural questions: a benchmark for question answering research. Transactions of the Association for Computational Linguistics (TACL).

Zhenzhong Lan, Mingda Chen, Sebastian Goodman, Kevin Gimpel, Piyush Sharma, and Radu Soricut. 2020. Albert: A lite bert for self-supervised learning of language representations. In International Conference on Learning Representations (ICLR).

Juho Lee, Yoonho Lee, Jungtaek Kim, Adam Kosiorek, Seungjin Choi, and Yee Whye Teh. 2019. Set transformer: A framework for attention-based permutation-invariant neural networks. In International Conference on Machine Learning (ICML).

Mike Lewis, Yinhan Liu, Naman Goyal, Marjan Ghazvininejad, Abdelrahman Mohamed, Omer Levy, Ves Stoyanov, and Luke Zettlemoyer. 2019. Bart: Denoising sequence-to-sequence pre-training for natural language generation, translation, and comprehension. In Association for Computational Linguistics $(A C L)$.

Yankai Lin, Haozhe Ji, Zhiyuan Liu, and Maosong Sun. 2018. Denoising distantly supervised open-domain question answering. In Association for Computational Linguistics (ACL).

Dayiheng Liu, Yeyun Gong, Jie Fu, Yu Yan, Jiusheng Chen, Daxin Jiang, Jiancheng Lv, and Nan Duan. 2020. Rikinet: Reading wikipedia pages for natural question answering. In Association for Computational Linguistics $(A C L)$.

Yinhan Liu, Myle Ott, Naman Goyal, Jingfei Du, Mandar Joshi, Danqi Chen, Omer Levy, Mike Lewis, Luke Zettlemoyer, and Veselin Stoyanov. 2019. Roberta: A robustly optimized bert pretraining approach. arXiv preprint arXiv: 1907.11692.

Matt Mahoney. 2011. Large text compression benchmark. URL: http://www. mattmahoney. net/text/text. html.

Stephen Merity, Caiming Xiong, James Bradbury, and Richard Socher. 2017. Pointer sentinel mixture models. In International Conference on Learning Representations (ICLR).

Lin Pan, Rishav Chakravarti, Anthony Ferritto, Michael Glass, Alfio Gliozzo, Salim Roukos, Radu Florian, and Avirup Sil. 2019. Frustratingly easy natural question answering. arXiv preprint arXiv:1909.05286.

Jack W Rae, Anna Potapenko, Siddhant M Jayakumar, and Timothy P Lillicrap. 2019. Compressive transformers for long-range sequence modelling. In International Conference on Learning Representations (ICLR).

Aurko Roy, Mohammad Saffar, Ashish Vaswani, and David Grangier. 2020. Efficient content-based sparse attention with routing transformers. Transactions of the Association for Computational Linguistics (TACL).

Yi Tay, Dara Bahri, Donald Metzler, Da-Cheng Juan, Zhe Zhao, and Che Zheng. 2021. Synthesizer: Rethinking self-attention in transformer models. In International Conference on Machine Learning $(I C M L)$

Yi Tay, Dara Bahri, Liu Yang, Donald Metzler, and Da-Cheng Juan. 2020a. Sparse sinkhorn attention. In International Conference on Machine Learning (ICML).

Yi Tay, Mostafa Dehghani, Dara Bahri, and Donald Metzler. 2020b. Efficient transformers: A survey. arXiv preprint arXiv:2009.06732.

Yi Tay, Anh Tuan Luu, Siu Cheung Hui, and Jian Su. 2018. Densely connected attention propagation for reading comprehension. In Advances in Neural Information Processing Systems (NeurIPS).

Ashish Vaswani, Noam Shazeer, Niki Parmar, Jakob Uszkoreit, Llion Jones, Aidan N Gomez, Łukasz Kaiser, and Illia Polosukhin. 2017. Attention is all you need. In Advances in Neural Information Processing Systems (NeurIPS).

Apoorv Vyas, Angelos Katharopoulos, and François Fleuret. 2020. Fast transformers with clustered attention. Advances in Neural Information Processing Systems (NeurIPS).

Shuohang Wang, Mo Yu, Xiaoxiao Guo, Zhiguo Wang, Tim Klinger, Wei Zhang, Shiyu Chang, Gerry Tesauro, Bowen Zhou, and Jing Jiang. 2018. R3: Reinforced ranker-reader for open-domain question answering. In $A A A I$ Conference on Artificial Intelligence $(A A A I)$.

Sinong Wang, Belinda Li, Madian Khabsa, Han Fang, and Hao Ma. 2020. Linformer: Selfattention with linear complexity. arXiv preprint arXiv:2006.04768.

Zhiguo Wang, Patrick Ng, Xiaofei Ma, Ramesh Nallapati, and Bing Xiang. 2019. Multi-passage bert: A globally normalized bert model for open-domain question answering. In Empirical Methods in Natural Language Processing (EMNLP). 
Manzil Zaheer, Guru Guruganesh, Avinava Dubey, Joshua Ainslie, Chris Alberti, Santiago Ontanon, Philip Pham, Anirudh Ravula, Qifan Wang, Li Yang, et al. 2020. Big bird: Transformers for longer sequences. In Advances in Neural Information Processing Systems (NeurIPS).

Junru Zhou and Hai Zhao. 2019. Head-driven phrase structure grammar parsing on penn treebank. In $A s$ sociation for Computational Linguistics (ACL). 